\title{
Gas Dispersion in Highly Viscous Fluids with a Coaxial Mixer through Tomography and CFD
}

\author{
Farhad Ein-Mozaffari ${ }^{1}$, Nasim Hashemi ${ }^{2}$ \\ ${ }^{1}$ Department of Chemical Engineering, Ryerson University \\ 350 Victoria Street, Toronto, ON., M5B 2K3, Canada \\ fmozaffa@ryerson.ca \\ ${ }^{2}$ Department of Chemical Engineering, Ryerson University \\ 350 Victoria Street, Toronto, ON., M5B 2K3, Canada \\ nasim.hashemi@ryerson.ca
}

\section{Extended Abstract}

The hydrodynamics of the gas-liquid flow in a bioreactor containing a highly viscous fluid was investigated using the non-invasive flow visualization technique called electrical resistance tomography (ERT) and computational fluid dynamics (CFD). The bioreactor was furnished with a coaxial mixer [1] composed a close clearance impeller (e.g. an anchor impeller) and a central impeller (e.g. a pitched blade turbine).

The dynamic gas disengagement theory coupled with the tomography data [2] was employed to determine the local and global gas holdups, number of bubble size classes, contribution of each class of bubbles, and the bubble sizes within the bioreactor. We also developed a computational fluid dynamics model (CFD) using the Eulerian-Eulerian method coupled with the population balance model to assess the behaviour of bubbles in the viscous fluids. To model the rotation of both impellers (i.e. the central impeller and the anchor impeller), the sliding mesh technique was used. The bubble size distribution within the aerated coaxial mixer was estimated using the Multiple Size Group (MUSIG) model including the bubble breakage and coalescence kernels. The Laakkonen breakage kernel [3] was utilized to simulate the breakage frequency. The coalescence rate was also determined through Abrahamson's model [4]. The modified Tomiyama model [5] based on the Brucato model was used to estimate the drag force.

To validate the CFD model, the simulated Sauter mean bubble diameters were compared with the experimental values obtained from the tomography images. In this study, the tomography data and the validated CFD model were employed to assess the effects of the speed ratio (central impeller speed / anchor speed), rotating mode (co-rotating and counter-rotating modes), fluid viscosity, and gas flow rate on the gas holdup, mixing time, power consumption, and bubble size distribution.

The data obtained in this study, enabled us to develop two correlations for the gas flow number and power number. The results revealed that at the higher fluid viscosity, the anchor power consumption increased at the speed ratios higher than the critical value of 10 . The CFD results showed that the breakage was more pronounced at the bottom of the tank while the coalescence was more dominant at the top of the tank. It was found that at the speed ratio of 10, the volume fraction of the large bubbles decreased while the volume fraction of the small bubbles increased. The turbulent kinetic energy achieved in the counter-rotating mode was less than that attained in the co-rotating mode.

\section{References}

[1] N. Hashemi, F. Ein-Mozaffari, S. R. Upreti, and D. K. Hwang, "Analysis of mixing in an aerated reactor equipped with the coaxial mixer through electrical resistance tomography and response surface method," Chem. Eng. Res. Des., vol. 109, pp. 734-752, 2016.

[2] N. Hashemi, F. Ein-Mozaffari, S. R. Upreti, and D. K. Hwang, "Experimental investigation of the bubble behavior in an aerated coaxial mixing vessel through electrical resistance tomography (ERT)," Chem. Eng. J., vol. 289, pp. 402412, 2016.

[3] M. Laakkonen, V. lopaeus, and J. Aittamaa, "Validation of bubble breakage, coalescence and mass transfer models for gas-liquid dispersion in agitated vessel," Chem. Eng. Sci., vol. 61, pp. 218-228, 2006. 
[4] J. Abrahamson, "Collision rates of small particles in a vigorously turbulent fluid," Chem. Eng. Sci., vol. 30, pp. 13711379, 1975.

[5] A. Tomiyama, "Struggle with computational bubble dynamics," Multiphase Sci. and Technol., vol. 10, pp. 369-405, 1998. 